Mon. Not. R. Astron. Soc. 000,113(2021) Printed 11 January $2022 \quad$ (MN LATEX style file v2.2)

\title{
V4334 Sgr (Sakurai's Object): still churning out the dust
}

\author{
A. Evans $^{1 \star}$, D. P. K. Banerjee ${ }^{2}$, T. R. Geballe ${ }^{3}$, R. D. Gehrz ${ }^{4}$, C. E. Woodward ${ }^{4} \dagger$, \\ K. Hinkle ${ }^{5}$, R. R. Joyce ${ }^{5}$, M. Shahbandeh ${ }^{6}$ \\ ${ }^{1}$ Astrophysics Group, Keele University, Keele, Staffordshire, ST5 5BG, UK \\ ${ }^{2}$ Physical Research Laboratory, Ahmedabad 380009, India \\ ${ }^{3}$ Gemini Observatory/NSF's NOIRLab, 670 N. A'ohoku Place, Hilo, Hawai'i, 96720, USA \\ ${ }^{4}$ Minnesota Institute for Astrophysics, School of Physics 83 Astronomy, 116 Church Street SE, University of Minnesota, \\ Minneapolis, MN 55455, USA \\ ${ }^{5}$ National Optical-Infrared Astronomy Research Laboratory, 950 N. Cherry Avenue, Tucson, AZ 85719, USA \\ ${ }^{6}$ Department of Physics, Florida State University, 77 Chieftain Way, Tallahassee, FL 32306-4350, USA
}

Version of 11 January 2022

\begin{abstract}
We present a $0.8-2.5 \mu \mathrm{m}$ spectrum of the Very Late Thermal Pulse object V4334 Sgr (Sakurai's Object), obtained in 2020 September. The spectrum displays a continuum that rises strongly to longer wavelengths, and is considerably brighter than the most recent published spectrum obtained seven years earlier. At the longer wavelengths the continuum is well fitted by a blackbody with a temperature of $624 \pm 8 \mathrm{~K}$. However, there is excess continuum at the shortest wavelengths that we interpret as being due to hot dust that has very recently formed in an environment with $\mathrm{C} / \mathrm{O} \simeq 2.5$. Other possible sources for this excess continuum are discussed - such as the stellar photosphere dimly seen through the dust shell, and light scattered off the inner wall of the dust torus but these interpretations seem unlikely. Numerous emission lines are present, including those of HeI, C I, [C I], and O I. Our observations confirm that emission in the He I $1.083 \mu \mathrm{m}$ and [C I] $0.9827 / 0.9852 \mu \mathrm{m}$ lines is spatially extended. The [C I] line fluxes suggest that the electron density increased by an order of magnitude between 2013 and 2020, and that these two lines may soon disappear from the spectrum. The flux ratio of the $1.083 \mu \mathrm{m}$ and $2.058 \mu \mathrm{m}$ He I lines is consistent with the previously-assumed interstellar extinction. The stellar photosphere remains elusive, and the central star may not be as hot as suggested by current evolutionary models.
\end{abstract}

Key words: stars: AGB and post-AGB - stars: carbon - circumstellar matter stars: evolution - stars: individual, V4334 Sgr (Sakurai's Object) - infrared: stars

\section{INTRODUCTION}

V4334 Sgr (Sakurai's Object; hereafter SO) has been widely considered to be the product of a Very Late Thermal Pulse $(\mathrm{VLTP})$ in a low $(\sim$ solar $)$ mass star. The star became carbon-rich in mid-1996 (Eyres et al. 1998), and in late 1997 it ejected a carbon-rich dust shell that became optically thick in mid-1998; by the end of that year the dust had completely obscured the central star (see Figure 2 of Dürbeck et al. 2000).

As of late 2021, the star has not reappeared. However, observations during the last $1 \frac{1}{2}$ decades van Hoof et al.

\footnotetext{
* E-mail: a.evans@keele.ac.uk

$\dagger$ Visiting Astronomer at the Infrared Telescope Facility, which is operated by the University of Hawaii under contract 80HQTR19D0030 with the National Aeronautics and Space Administration.
}

2007, 2008; Hinkle \& Jovce 2014; van Hoof et al. 2015a, b, 2018; Hinkle et al. 2020) have shown that the optical and near-infrared (NIR) spectra originating inside the dust shell are starting to become detectable.

A comprehensive account of the infrared (IR) emission from SO was given by Evans et al. 2020), who also reviewed determinations of the distance and interstellar reddening; we assume their values here, namely $D=3.8 \mathrm{kpc}$ and $E(B-V)=0.62$. Chesneau et al. (2009) showed that the dust around SO takes the form of a disc/torus (hereafter "disc"). The presence of an optically thick disc, the opacity of which is unknown, clearly renders the determination of the total (interstellar plus circumstellar) reddening somewhat uncertain. This is discussed below.

Here we present a $0.8-2.5 \mu \mathrm{m}$ spectrum of SO, obtained at the Frederick C. Gillett Gemini North Telescope. The new spectrum indicates that the trends reported since 2007 are continuing. 


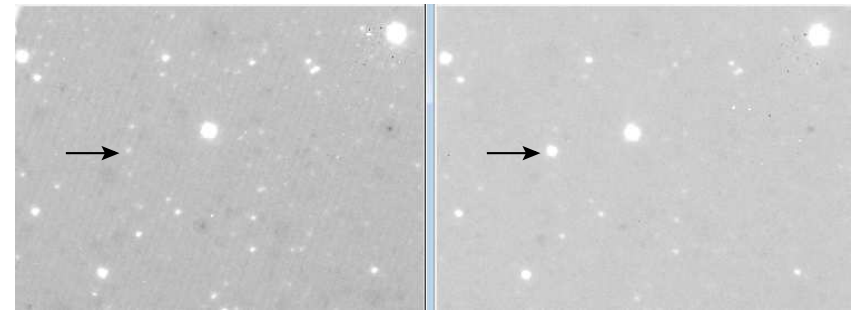

Figure 1. $J K$ images of SO obtained on the $3.2 \mathrm{~m}$ NASA Infrared Telescope Facility on 2020 June 07.46 UT. Left: J-band image, right: $K$-band image. Scale is $\sim 45^{\prime \prime} \times 30^{\prime \prime}$. North is up, east is left. SO is arrowed.
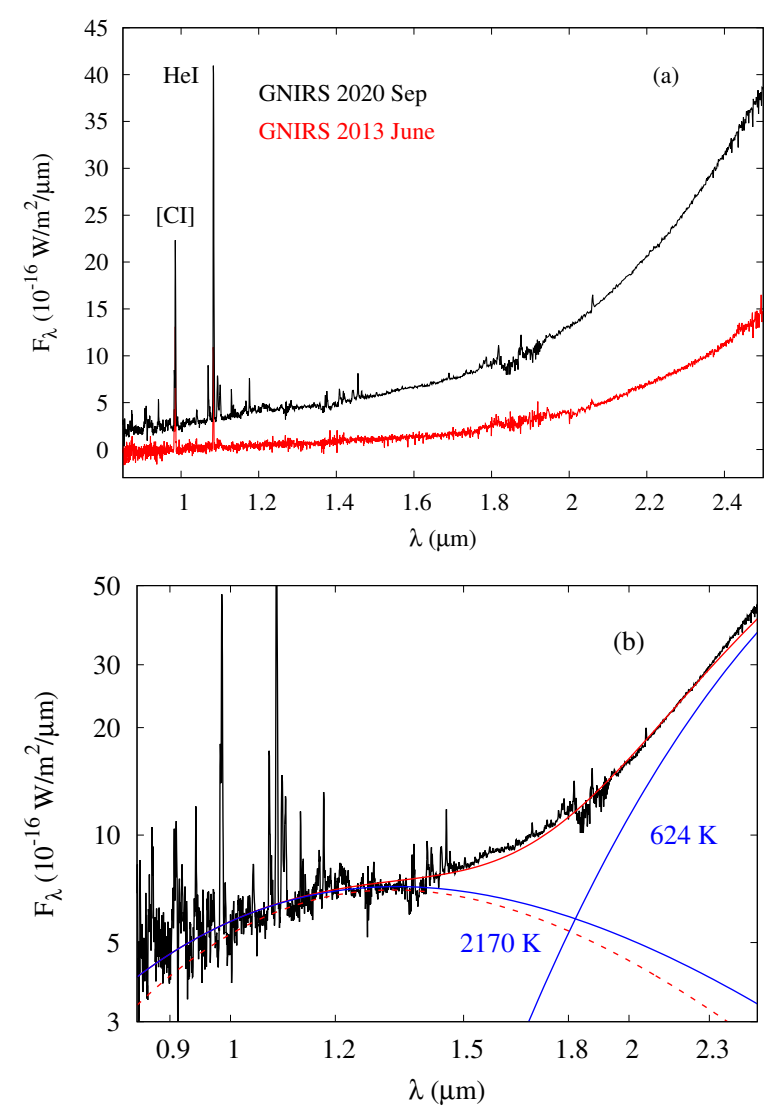

Figure 2. (a) Comparison of the observed 2013 June spectrum (red; Hinkle \& Joyce 2014) and the 2020 September spectrum (black). (b) The 2020 spectrum (black), dereddened by $E(B-$ $V)=0.62$; wavelength scale is logarithmic to stretch the scale at the shortest wavelengths. The full red curve is a two-blackbody fit to the continuum; the blue curves are the individual blackbodies at the temperatures indicated. The dotted red curve is a $10^{4} \mathrm{~L} \odot$, $8 \times 10^{4} \mathrm{~K}$ blackbody, reddened by $A_{V}=9.3 \mathrm{mag}$, as described in the text.

\section{OBSERVATIONS AND DATA REDUCTION}

Spectra of SO and a telluric standard, HIP 90404 (A0V), were obtained on UT 2020 September 29 for programme GN-2020B-FT-206. The facility NIR spectrograph GNIRS (Elias et al. 2006) was used in its cross-dispersed mode with a 0 .' $45 \times 7$ ". 0 slit, yielding a spectral resolving power, $R$, of $\sim 1200$ across the $1.0-2.5 \mu \mathrm{m}$ band, and slightly lower $R$ at shorter wavelengths. The standard nod-along slit (ABBA) mode was used with a nod of $\pm 1^{\prime \prime} .5$ from slit centre. The slit was oriented at a position angle of $35^{\circ}$ east of north. The total exposure time on SO was 2400 seconds. SO and the telluric standard were observed at airmasses within several hundredths of 1.4. The spectra were obtained in clear skies with good and stable seeing. Measurements of the spectral images yield full widths at half maxima of 0.57 and 0.51 , at $1.0 \mu \mathrm{m}$ and $2.2 \mu \mathrm{m}$, respectively.

Data reduction with the Gemini IRAF (Tody 1986) and Starlink FIGARO Shortridge et al. 2004) packages involved the standard steps of flat-fielding, spatial and spectral rectification of the images, removal of the effects of cosmic ray hits, order by order extraction of the spectra, spectral cross correlation and combining of the negative and positive spectra, and wavelength calibration using an argon lamp. Following the removal of most of the photospheric hydrogen recombination lines in the spectrum of the telluric standard and wavelength alignment of the SO and telluric standard, flux calibration was achieved by ratioing the two spectra under the assumption that HIP 90404 has the continuum of a $9480 \mathrm{~K}$ blackbody and the JHK magnitudes listed in SIM$\mathrm{BAD}^{1}$. We estimate that the flux calibration is accurate to $\pm 20 \%$.

The $J$ and $K$ band images shown in Fig. 1 were obtained with the SpeX (Rayner et al. 2003) guider imager on the NASA Infrared Telescope Facility on 2020 June 07.46 $\mathrm{UT}$, using a five point dither with $40 \mathrm{~s}$ exposure time at each position, and at an average airmass of 1.26. Data reduction was done in a standard manner, involving sky and dark subtraction, and flatfielding. Instrumental magnitudes obtained through aperture photometry were calibrated against several 2MASS field stars; we find $J=16.89 \pm 0.09 \mathrm{mag}$, $K_{s}=12.66 \pm 0.22 \mathrm{mag}$ for $\mathrm{SO}$ at this epoch.

\section{RESULTS AND DISCUSSION}

The observed spectrum, extracted over a $1^{\prime \prime}$ region of the slit centred on the continuum peak, is shown in Fig. 2a, along with the spectrum obtained by Hinkle \& Joyce (2014) with GNIRS on 2013 June 14. The comparison indicates that significant changes occurred in the intervening $\sim 7$ years. In our spectrum, the apparent emission features around $1.8-1.9 \mu \mathrm{m}$ are due to incomplete removal of the hydrogen absorption features in the spectrum of the telluric standard.

Fig. 20 shows the new spectrum, along with blackbody fits to the continuum, which are discussed in Section 3.1 Numerous emission lines are present, especially in the 0.8 $1.7 \mu \mathrm{m}$ region. This spectral interval is shown in more detail in Fig. 3, where most of the lines are identified.

\subsection{The continuum}

As is evident from Fig. 2a,b, the flux density was rising from $\sim 1.4 \mu \mathrm{m}$ to longer wavelengths in 2013 and 2020, indicating emission by dust. In this respect the 2013 and 2020 spectra are broadly similar. However there are two key differences: First, in 2020 there is an additional excess above 


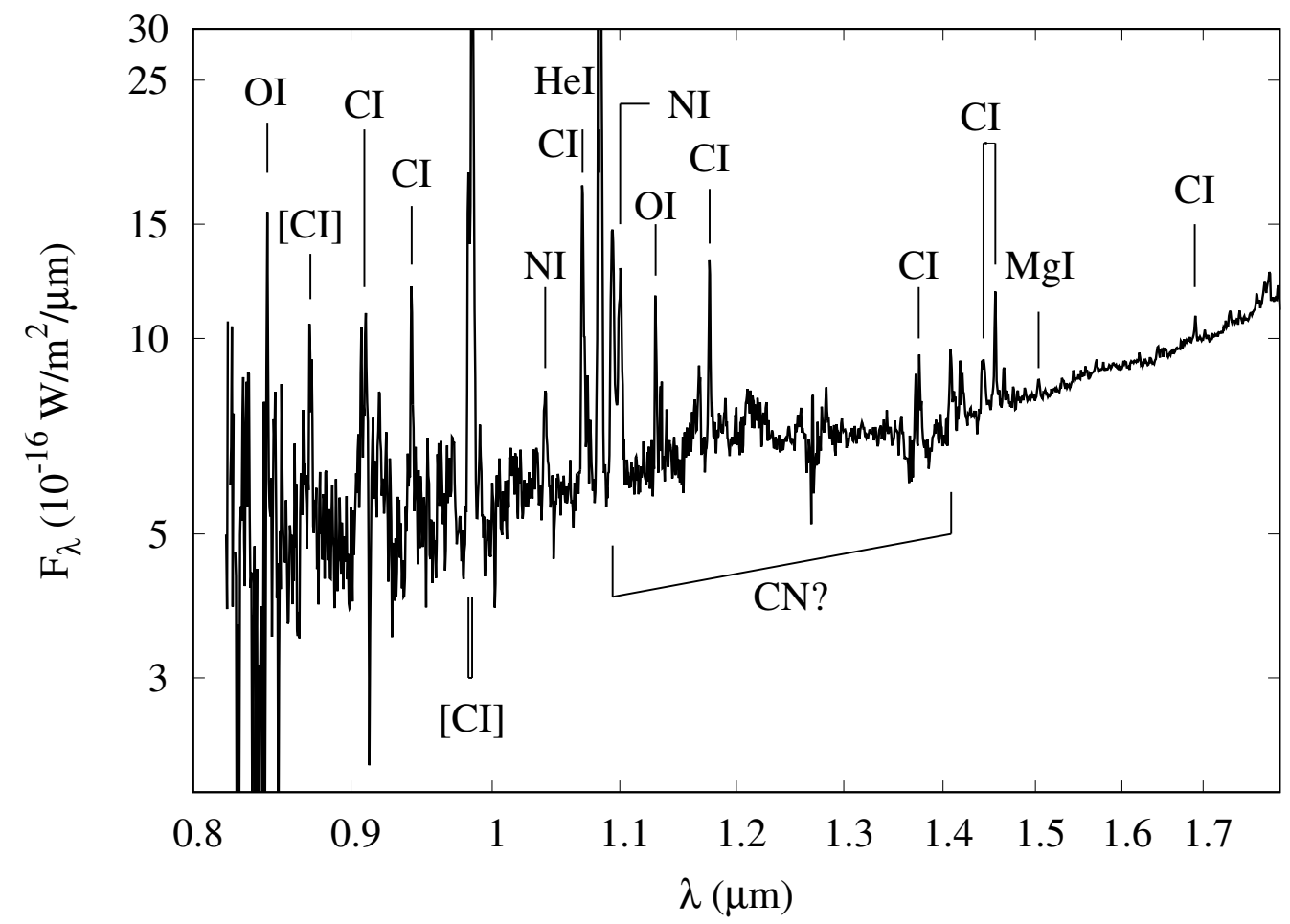

Figure 3. Identification of emission lines in the 2020 September spectrum; see also Table 1 and Fig. 7 Wavelength scale is logarithmic to stretch the scale at the shortest wavelengths. The small dips in the apparent continuum near $1.27 \mu \mathrm{m}$ and in the $1.35-1.40 \mu \mathrm{m}$ interval are due to incomplete cancellation of strong telluric $\mathrm{O}$ and $\mathrm{H}_{2} \mathrm{O}$ absorption bands, respectively. The emission features in that interval are real and intrinsic to SO.

the dust continuum at the shortest wavelengths: no continuum around $1 \mu \mathrm{m}$ was detected by Hinkle \& Joyce (2014); its contribution was $\lesssim 10^{-16} \mathrm{~W} \mathrm{~m}^{-2} \mu \mathrm{m}^{-1}$ at $1.4 \mu \mathrm{m}$. Second, the continuum flux density was considerably greater at all wavelengths in 2020; for example it was three times higher at $2.4 \mu \mathrm{m}$.

The spectra and photometry indicate a steady rise in the flux in the $K_{s}$ band, from $K_{s}>18.4$ in 2009 March, to $K_{s} \simeq 14$ around the time of the Hinkle \& Jovce observation, to $13.20 \pm 0.02$ in 2017 October (see Table 2 and Figure 3 in Evans et al. 2020), to $K_{s}=12.66 \pm 0.22$, close to the time of the 2020 September GNIRS observation.

We assume that the NIR continuum is subject to interstellar extinction only; we briefly explore the effects of internal extinction below. A fit of two blackbodies to the continuum from the 2020 spectrum, dereddened by $E(B-V)=$ 0.62 (Evans et al. 2020, see also below), gives temperatures of $624 \pm 8 \mathrm{~K}$ ("Component 1") and $2170 \pm 25 \mathrm{~K}$ ("Component 2") for the two components (see Fig. 2p). Component 1 is clearly due to emission by dust. Assuming that the dominant dust component is amorphous carbon (AC; see Evans et al. 2020, and references therein for a justification of this), we deduce a dust mass of $5.7[ \pm 1.6] \times 10^{-10} \mathrm{M}_{\odot}$ and a bolometric dust luminosty of $38[ \pm 10] \mathrm{L}_{\odot}$ for a distance $D=3.8 \mathrm{kpc}$.

Hinkle \& Jovce 2014) determined that, in 2013, the dust continuum (corresponding to our Component 1) had a temperature of $590 \mathrm{~K}$; a recalculation using the same procedure as that used here (i.e. same dereddening, same blackbody fit), for consistency, yields $550 \pm 40 \mathrm{~K}$; the correspond- ing dust mass and luminosity are $8.5[ \pm 3.8] \times 10^{-10} \mathrm{M}_{\odot}$ and $31 \mathrm{~L} \odot$ respectively. There is no significant difference between the dust masses in 2013 and 2020.

The interpretation of Component 2 is not as straightforward. The value of $\left[\lambda f_{\lambda}\right]_{\max }$ for this component is $1.07 \times$ $10^{-15} \mathrm{~W} \mathrm{~m}^{-2}$, or $\sim 0.5 \mathrm{~L}_{\odot}$ at $3.8 \mathrm{kpc}$. There appear to be three possibilities, which we discuss below: (a) the stellar photosphere is being viewed, through several magnitudes of visual circumstellar extinction, (b) light from an embedded stellar remnant is scattered off the inner wall of the dust disc, (c) the emission is from extremely hot, very recently-formed dust.

(a) The stellar photosphere. Evolutionary models (e.g., Hajduk et al. 2005) suggest that, in its current $(\sim 2021.0)$ state, SO should have had an effective temperature $T_{*} \sim$ $10^{5} \mathrm{~K}$ and bolometric luminosity $L_{*} \sim 10^{3.8} \mathrm{~L} \odot$. Such a source, at a distance of $3.8 \mathrm{kpc}$, would have $\left[\lambda f_{\lambda}\right]_{\max } \simeq$ $2.2 \times 10^{-11} \mathrm{~W} \mathrm{~m}^{-2}$, but would be seen through a large (but unknown) amount of circumstellar extinction and reddening (parametrised by the visual extinction, $A_{V}$, in magnitudes). A reddening law in which $A_{\lambda}$ is approximately proportional to $\lambda^{-1}$ has the effect of making a blackbody appear cooler than it actually is, and for large reddening a $10^{5} \mathrm{~K}$ blackbody can appear as cool as $\sim 3000 \mathrm{~K}$. 
Table 1. Emission lines in the spectrum of SO; the spectrum was extracted over a $1^{\prime \prime}$ region of the slit, centered on the continuum peak. $\lambda_{\text {obs }}$ and $\lambda_{\text {id }}$ are the measured and listed vacuum swavelengths respectively, the latter from van Hoof (2018) unless specified otherwise. Dereddening in the penultimate column is by $E(B-V)=0.62$.

\begin{tabular}{|c|c|c|c|c|c|c|}
\hline$\lambda_{\text {obs }}(\mu \mathrm{m})$ & Identification & $\lambda_{\mathrm{id}}(\mu \mathrm{m})^{*}$ & $\begin{array}{l}\text { Transition } \\
u-\ell\end{array}$ & $\frac{\text { Line flux }(1}{\text { Observed }}$ & $\frac{\left.-18 \mathrm{~W} \mathrm{~m}^{-2}\right)}{\text { Dereddened }}$ & Comment \\
\hline 0.8455 & O I & 0.8448 & ${ }^{3} \mathrm{P}-{ }^{3} \mathrm{~S}^{o}$ & $0.45 \pm 0.04$ & $1.11 \pm 0.10$ & क \\
\hline 0.8732 & {$[\mathrm{C} \mathrm{I}]$} & 0.8729 & ${ }^{1} \mathrm{~S}_{0}-{ }^{1} \mathrm{D}_{2}$ & $0.44 \pm 0.05$ & $1.09 \pm 0.14$ & Wavelength from Haris \& Kramida (2017). $\stackrel{\overbrace{}}{\sim}$ \\
\hline 0.9090 & $\mathrm{C}_{\mathrm{I}}$ & $0.9094-0.9097$ & ${ }^{3} \mathrm{P}-{ }^{3} \mathrm{P}^{o}$ & $1.13 \pm 0.13$ & $2.32 \pm 0.25$ & Blend of 5 lines. \\
\hline 0.9417 & $\mathrm{C}_{\mathrm{I}}$ & 0.9408 & ${ }^{1} \mathrm{D}_{2}-{ }^{1} \mathrm{P}_{2}^{o}$ & $0.50 \pm 0.05$ & $1.03 \pm 0.12$ & \\
\hline 0.9825 & {$[\mathrm{C} \mathrm{I}]$} & 0.9824 & ${ }^{1} \mathrm{D}_{2}-{ }^{3} \mathrm{P}_{1}^{2}$ & $0.90 \pm 0.05$ & $1.92 \pm 0.12$ & $\begin{array}{l}\text { Wavelength from Haris \& Kramida (2017). } \\
\text { Detected by Hinkle \& Jovce (2014). }\end{array}$ \\
\hline 0.9852 & {$\left[\mathrm{C}_{\mathrm{I}}\right]$} & 0.9850 & ${ }^{1} \mathrm{D}_{2}-{ }^{3} \mathrm{P}_{2}$ & $4.00 \pm 0.07$ & $8.45 \pm 0.16$ & $\begin{array}{l}\text { Wavelength from Haris \& Kramida } \\
\text { Detected by Hinkle \& Joyce (2014) }\end{array}$ \\
\hline 1.0405 & $\mathrm{~N}_{\mathrm{I}}$ & $\begin{array}{l}1.0405 \\
1.0411\end{array}$ & $\begin{array}{l}{ }^{2} \mathrm{D}_{3 / 2}-{ }^{2} \mathrm{D}_{5 / 2}^{o} \\
{ }^{2} \mathrm{D}_{5 / 2}-{ }^{2} \mathrm{D}_{5 / 2}^{o}\end{array}$ & $0.30 \pm 0.03$ & $0.63 \pm 0.05$ & Detected by Hinkle \& Jovce (2014). \\
\hline 1.0698 & $\mathrm{C}_{\mathrm{I}}$ & 1.0688 & ${ }^{3} \mathrm{D}-{ }^{3} \mathrm{P}^{o}$ & $1.29 \pm 0.13$ & $2.47 \pm 0.09$ & \\
\hline 1.0835 & He I & 1.0833 & ${ }^{3} \mathrm{P}^{o}-{ }^{3} \mathrm{~S}$ & $8.94 \pm 0.12$ & $16.88 \pm 0.24$ & Detected by Hinkle \& Jovce (2014). \\
\hline 1.0940 & CN? & & & $1.53 \pm 0.14$ & $2.86 \pm 0.07$ & $\begin{array}{l}\text { Detected by Hinkle \& Jovce (2014) but unidentified. } \\
\text { See Section 3.2.4 }\end{array}$ \\
\hline 1.1003 & $\mathrm{~N}_{\mathrm{I}}$ & 1.0992 & ${ }^{2} \mathrm{~F}_{7 / 2}-{ }^{2} \mathrm{~F}_{7 / 2}^{o}$ & $1.23 \pm 0.15$ & $2.31 \pm 0.07$ & Detected by Hinkle \& Joyce (2014) but unidentified. \\
\hline 1.1298 & O I & 1.1290 & ${ }^{3} \mathrm{D}_{3}^{o}-{ }^{3} \mathrm{P}_{2}$ & $0.44 \pm 0.03$ & $0.80 \pm 0.05$ & \\
\hline 1.1671 & $\mathrm{C}_{\mathrm{I}}$ & $1.1656-1.1677$ & & $0.34 \pm 0.05$ & $0.42 \pm 0.06$ & Blend of 6 lines. \\
\hline 1.1762 & $\mathrm{C}_{\mathrm{I}}$ & $1.1751-1.1781$ & & $0.65 \pm 0.03$ & $1.11 \pm 0.06$ & Blend of 4 lines. \\
\hline 1.3756 & $\mathrm{C}_{\mathrm{I}}$ & 1.3727 & ${ }^{1} \mathrm{~S}_{0}-{ }^{1} \mathrm{P}_{1}^{o}$ & $0.22 \pm 0.02$ & $0.34 \pm 0.04$ & \\
\hline 1.4085 & CN? & & & $0.36 \pm 0.04$ & $0.88 \pm 0.09$ & See Section 3.2.4 \\
\hline 1.4429 & $\mathrm{C}_{\mathrm{I}}$ & 1.4424 & ${ }^{3} \mathrm{D}_{3}^{o}-{ }^{3} \mathrm{P}_{2}$ & $0.43 \pm 0.04$ & $0.68 \pm 0.06$ & \\
\hline 1.4557 & $\mathrm{C}_{\mathrm{I}}$ & 1.4547 & ${ }^{1} \mathrm{P}_{1}-{ }^{1} \mathrm{P}_{1}^{o}$ & $0.54 \pm 0.03$ & $0.81 \pm 0.05$ & \\
\hline 1.4650 & $\mathrm{C}$ I] & 1.4641 & ${ }^{3} \mathrm{~F}_{3}-{ }^{3} \mathrm{P}_{2}^{o}$ & $0.15 \pm 0.03$ & $0.22 \pm 0.03$ & \\
\hline 1.5036 & Mg I & $1.5029-1.5052$ & ${ }^{3} \mathrm{~S}^{o}-{ }^{3} \mathrm{P}$ & $0.10 \pm 0.01$ & $0.17 \pm 0.02$ & Blend of 3 lines. \\
\hline 1.6902 & $\mathrm{C}_{\mathrm{I}}$ & 1.6895 & ${ }^{1} \mathrm{~F}_{3}^{o}-{ }^{1} \mathrm{D}_{2}$ & $0.19 \pm 0.02$ & $0.24 \pm 0.03$ & \\
\hline 2.0602 & He I & 2.0589 & ${ }^{3} \mathrm{P}^{o}-{ }^{3} \mathrm{~S}$ & $0.45 \pm 0.03$ & $0.53 \pm 0.04$ & $\begin{array}{l}\text { Detected by Hinkle \& Jovce (2014). } \\
\text { Weak P-Cygni profile? }\end{array}$ \\
\hline
\end{tabular}

*Where a line is identified as a "Blend" the range of wavelengths is given. 
We have explored the $\left[L_{*}, T_{*}, A_{V}\right]$ parameter space, and guided by the values in Haiduk et al. for $L_{*}$ and $T_{*}$ and reasonable values of $A_{V}$; for example, Tyne et al. (2002) determined that $A_{V}$ was in the range 8-12 in the period 1999-2001. The reddening law for the circumstellar dust is of course unknown; we assume that it is interstellar-like in the $0.8-2.6 \mu \mathrm{m}$ wavelength range. We find that a $L_{*}=10^{4} \mathrm{~L}_{\odot}$, $T_{*}=8 \times 10^{4} \mathrm{~K}$ blackbody, reddened by an amount corresponding to $A_{V}=9.3$, is satisfactorily similar to Component 2 (see Fig. 2 $\mathrm{b}$ ). However such a source would easily have been detected by Hinkle \& Joyce (2014) in 2013 June, even allowing for 7-year variations in the stellar and dust parameters. This interpretation seems therefore unlikely.

(b) Scattered light. Might Component 2 be due to light from the obscured star, scattered off the inner wall of the disc? Chesneau et al. (2009) show that the inclination of the disc is $\simeq 75^{\circ}$, and its large scale height $(\sim 50 \mathrm{au}$, scaled to $D=3.8 \mathrm{kpc})$ is such that it limits the opening angle at the "poles"; they also determine the dimensions of the disc which, scaled to $3.8 \mathrm{kpc}$, are $\simeq 115 \times 150 \mathrm{au}$.

The scattering of light of a star embedded in an IR reflection nebula has been considered by Holbrook \& Temi (1998) and Pendleton. Tielens \& Werner 1990), and we primarily use the latter approach here. The flux from Component 2 in 2020 was $\sim 10^{-15} \mathrm{~W} \mathrm{~m}^{-2} \mu \mathrm{m}^{-1}$. With a $0^{\prime \prime} 45$ slit, and possible extended emission over $\sim 1^{\prime \prime}$ (see Section 4 below), this corresponds to an intensity $I_{\lambda} \gtrsim 11.4 \times$ $10^{-5} \mathrm{~W} \mathrm{~m}^{-2} \mu \mathrm{m}^{-1} \mathrm{sr}^{-1}$, the lower limit arising because the extent of the scattered light may be less than that given in Section 4 Using the formulae in Holbrook \& Temi and Pendleton et al., we get

$$
\begin{aligned}
I_{\lambda} & =\left(\frac{L_{*}}{4 \pi \sigma T_{*}^{4}}\right) \frac{B_{\lambda}\left(T_{*}\right)}{r^{2}} \frac{\omega}{4} \Delta_{\text {thin }} \\
& =\left(\frac{L_{*}}{4 \pi \sigma T_{*}^{4}}\right) \frac{B_{\lambda}\left(T_{*}\right)}{r^{2}} \frac{\omega}{4} \Delta_{\text {thick }}
\end{aligned}
$$

for the optically thin and optically thick cases respectively, where "optically thin" and "optically thick" refer to the optical depth $\tau$ of the scattering layer. $L_{*}$ and $T_{*}$ are the luminosity and temperature of the illuminating star respectively, and $r \simeq 120$ au is the distance of the star from the scattering surface. $\Delta_{\text {thin }}=\tau / \cos \theta$ and $\Delta_{\text {thick }}=\cos \theta_{0} /\left(\cos \theta+\cos \theta_{0}\right)$, where $\theta\left(\theta_{0}\right)$ is the angle of scattering (incidence). We take optical constants for AC grains from Hanner (1988); the albedo $\omega \sim 0.019$ for $0.1 \mu \mathrm{m} \mathrm{AC}$ grains at $1.3 \mu \mathrm{m}$ (the wavelength of maximum intensity).

The luminosity of the cooler dust seen in SOFIA data in mid-2016 was $\sim 3000 \mathrm{~L}_{\odot}$ (Evans et al. 2020). There are no long wavelength data later than 2016 but the timedependence of the dust luminosity (Evans et al. 2020) suggests that it would have been of this order around the time of the 2013 and 2020 NIR spectra. The $3000 \mathrm{~L}_{\odot}$ component must be radiation from the central star, reprocessed by the circumstellar dust; this value therefore serves as an estimate of the luminosity of the embedded star.

We have no value for $T_{*}$, but the function $B_{\lambda}\left(T_{*}\right) / T_{*}^{4}$ has a maximum value of $9.98 \times 10^{-9} \mathrm{~W} \mathrm{~m}^{-2} \mu \mathrm{m}^{-1} \mathrm{~K}^{-4}$ at $1.3 \mu \mathrm{m}$, irrespective of the value of $T_{*}$. Combining this with the lower limit on $I_{\lambda}$, we obtain a lower limit $\Delta \gtrsim 5.2 \times 10^{-4}$. We have no information about the scattering geometry, or of the optical depth $\tau$ to the scattered light through the dust shell. But surely this lower limit on $\Delta$ can be satisfied by a wide range of scattering geometries and optical depths. If Component 2 is scattered light then its intensity seems consistent with the presence of an embedded source, of unspecified temperature.

However Component 2 was not present in 2013. From Equation (11), the scattered intensity is $\propto L_{*}$. The dimensions of the disc are such that any scattered light would, by virtue of the finite speed of light, lag behind variations in the star by $\sim 1.5$ days, so the stellar and scattered light variations would essentially be in phase. The absence of Component 2 in 2013 would therefore have to be because either (i) the central star was much fainter in 2013 than it was in 2020 , by a factor $\gtrsim 10$ (the flux ratio $2020 / 2013$ ), (ii) the light reaching the scattering surface from the (unchanged) star was reduced by internal extinction, or (iii) the scattered light is itself obscured by internal extinction en route to the observer.

Case (i) would require that the luminosity of the star in 2013 would need to have been $\lesssim 300 \mathrm{~L}_{\odot}$, but the dust luminosity in 2013 is known to be $\gtrsim 3500 \mathrm{~L}_{\odot}$ Evans et al. 2020), which sets a lower limit on the luminosity of the embedded star. This inconsistency would lead us to conclude that this interpetation is unlikely.

However, given SO's proclivity for continuous dust ejection (Evans et al. 2020), it it not inconceivable that a discrete dust cloud (such as those described by Hinkle et al. 2020) happened to have been ejected around 2013 - away from our line-of-sight — so that light from the central star was prevented from reaching the scattering surface. Therefore, the observed scattered light would correspondingly be reduced. This behaviour is similar to that of the $\mathrm{R}$ Coronae Borealis stars during their well-known "dust dips" Clavton 2012). Such a cloud might also obscure the scattered light even if the star itself is visible from the scattering surface. These two scenarios refer respectively to cases (ii) and (iii) above.

In case (ii), the cloud must completely hide the star from the scattering surface, and its dimensions perpendicular to the light path must therefore be comparable with the disc scale height.

We assume a spherical cloud of radius $\ell$, where $\ell$ is a few au, and estimate the likely properties of the putative dust cloud as follows. Comparing the 2013 and 2020 fluxes at $1.25 \mu \mathrm{m}$ (where there are no prominent spectral features) we estimate the optical depth in the cloud to be $\tau_{1.25} \simeq$ 2.1 , assuming that the underlying continuum source has not varied between 2013 and 2020. Taking optical constants for AC grains from Hanner (1988), we determine the extinction efficiency $Q_{\text {ext }}$ at $1.25 \mu \mathrm{m}$ to be 0.253 . The optical depth, together with the extinction efficiency, gives the number of grains per unit volume, $n$, in the cloud as

$\left.n=\tau_{1.25} /\left[\pi a^{2} Q_{\text {ext }} 2 \ell\right]\right]$,

where $a$ is the grain radius and $2 \ell$ is the path length through the cloud.

The mass of dust in the obscuring cloud is

$$
\begin{aligned}
M_{\text {dust }} & \sim n \frac{4 \pi \ell^{3}}{3} \frac{4 \pi a^{3} \rho}{3}=\frac{8 \pi a \rho}{9} \ell^{2} \frac{\tau_{1.25}}{Q_{\mathrm{ext}}} \\
& \simeq 4.0 \times 10^{-11}\left(\frac{\ell}{\mathrm{au}}\right)^{2}\left(\frac{a}{0.1 \mu \mathrm{m}}\right) \mathrm{M}_{\odot},
\end{aligned}
$$


where $\rho$ is the density of the grain material (taken here to be $1.5 \mathrm{gm} \mathrm{cm}^{-3}$, the value assumed in Evans et al. 2020, although it transpires that the crucial result is independent of $\rho$ ). The temperature of the dust in the cloud, assuming $\mathrm{AC}$ and the data in Appendix B1 of Evans et al. (2017), is

$T_{\text {dust }}(\mathrm{K}) \simeq 540\left\{\left(\frac{L_{*}}{3350 \mathrm{~L}_{\odot}}\right)\left(\frac{a}{0.1 \mu \mathrm{m}}\right)\right\}^{1 /(\beta+4)}$

for a grain that is half-way between the star and the scattering surface. The parameter $\beta$ is defined such that the dust emissivity is $Q_{\mathrm{abs}} \propto \lambda^{-\beta}$; for $\mathrm{AC}, \beta=0.754$ (see Evans et al. 2017). The reference value of $L_{*}=3350 \mathrm{~L}_{\odot}$ is from Evans et al. (2020) for 2014, the nearest datum to 2013. Radiation from such a cloud would have $\left[\lambda f_{\lambda}\right]_{\max }$ at $\sim 6.8 \mu \mathrm{m}$.

The dust mass can be converted to $\left[\lambda f_{\lambda}\right]_{\max }$ for the obscuring dust cloud using the formulae in Evans et al. (2017). The quantity $\left[\lambda f_{\lambda}\right]_{\max }$ is independent of grain size, temperature and density:

$$
\left[\lambda f_{\lambda}\right]_{\max }=\frac{L_{*}}{32.62 \pi^{2} D^{2}} \frac{\tau_{1.25}}{Q_{\mathrm{ext}}}\left(\frac{\ell}{r}\right)^{2}
$$

Thus

$$
\left[\lambda f_{\lambda}\right]_{\max } \simeq 2.45 \times 10^{-12}\left(\frac{L_{*}}{3350 \mathrm{~L} \odot}\right)\left(\frac{\ell}{1 \mathrm{au}}\right)^{2}\left(\frac{r}{\mathrm{au}}\right)^{-2}
$$

where $\left[\lambda f_{\lambda}\right]_{\max }$ is in $\mathrm{W} \mathrm{m}^{-2}$.

Even a modest $\ell=5$ au ( $\ll$ the disc scale height) and $r \simeq 50$ au (about half-way from the star to the scattering surface) gives $\left[\lambda f_{\lambda}\right]_{\max } \simeq 2.44 \times 10^{-14} \mathrm{~W} \mathrm{~m}^{-2}$, significantly larger than the observed $1.07 \times 10^{-15} \mathrm{~W} \mathrm{~m}^{-2}$ for Component 2. Hence, emission from the dust cloud would overwhelm the fluxes in Fig. 2. The same conclusion applies to a dust cloud that obscures the scattered (as opposed to the direct) light, although in this case the dust temperature would be somewhat cooler.

We argue that Component 2 in 2020, and its absence in 2013, was the result of neither an intrinsic fading of the star (case (i)), the extinguished starlight scattered off the inner wall of the disc (case (ii)), nor the effect of a dust cloud preventing scattered starlight from reaching the observer (case (iii)).

(c) Hot dust. We consider whether the component with blackbody temperature $2170 \mathrm{~K}$ is extremely hot, freshlyformed, dust. While this temperature may appear high for dust, it is not unusual for carbon dust. For example Gall et al. (2014) found $T=2300 \mathrm{~K}$ on day 26 in supernova SN 2010jl, while hot dust has been found in novae (e.g. $\gtrsim 1400 \mathrm{~K}$ in V2362 Cyg (Lynch et al. 2008) 263 days after outburst, and $2000 \mathrm{~K}$ in V838 Her Harrisson \& Stringfellow 1994). Also, it is well-known that very small $(\sim 10 \AA)$ carbon grains (such as those that are newly formed) can attain very high temperatures by stochastic heating by single ultra-violet photons (Sellgren 1984). There was no silicon carbide feature at $11.5 \mu \mathrm{m}$ in the mid-IR spectrum of $\mathrm{SO}$ (Evans et al. 2020), so this hot component is likely to be carbonaceous rather than $\mathrm{SiC}$.

If, as seems probable, the dust is AC, then its actual temperature is somewhat less than $2170 \mathrm{~K}$. For a grain with $\beta=0.754$, the wavelength of maximum $f_{\lambda}$ is given by $\lambda_{\max } T=2890 \times 5 /(\beta+5) \mu \mathrm{m}-\mathrm{K}$ rather than

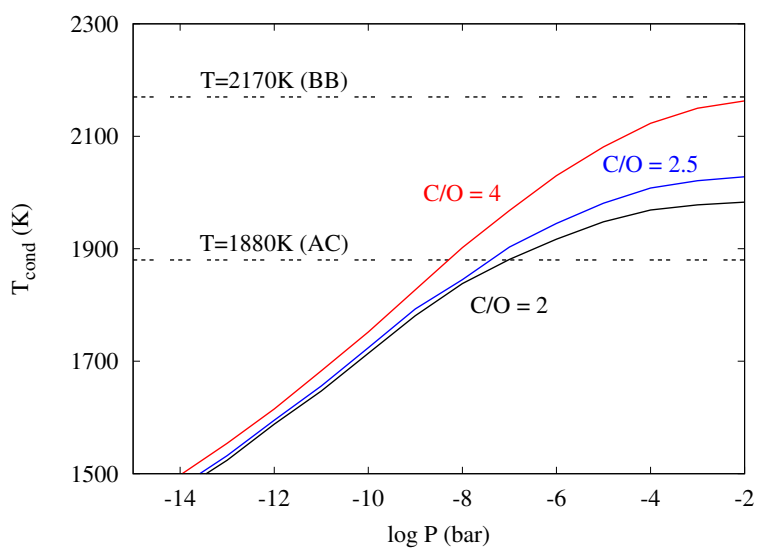

Figure 4. Condensation temperature for graphite grains for the values of $\mathrm{C} / \mathrm{O}$ indicated. Data from Table 2 of Lodders \& Fegley (1995). Data for blue curve $(\mathrm{C} / \mathrm{O}=2.5)$ have been linearly interpolated from Table 2 of Lodders \& Fegley. Dotted horizontal lines depict temperatures of hot blackbody and AC dust (Component 2), from Fig. 2p.

$\lambda_{\max } T=2890 \mu \mathrm{m}-\mathrm{K}$. The flux from Component 2 peaks at $\sim 1.3 \mu \mathrm{m}$, so that the dust temperature is $1880 \mathrm{~K}$. This is clearly in line with hot carbon dust seen in other sources.

While the stellar photosphere of SO was still visible, Asplund et al. (1999) determined the C/O ratio to be $\sim 2.5$. The condensation sequence in these conditions has been considered by Lodders \& Fegley (1995), who found that the condensation temperature for graphitic (as opposed to amorphous, as discussed here and in Evans et al. 2020) carbon in such an environment is very sensitive to the pressure. Their results for $\mathrm{C} / \mathrm{O}=2$ and 4 , together with our own linear interpolation for $\mathrm{C} / \mathrm{O}=2.5$, are shown in Fig. 4. Allowing for possible differences between the condensation conditions for $\mathrm{AC}$ and graphite, and for uncertainties in the $\mathrm{C} / \mathrm{O}$ ratio, it seems probable that $\mathrm{AC}$ could condense at $\sim 1880 \mathrm{~K}$ at pressure $\simeq 10^{-7.5}$ bar), requiring a density of carbon atoms $\simeq 1.2 \times 10^{6} \mathrm{~cm}^{-3}$.

The 2020 data, combined with those from 2013, suggest therefore that Component 2 represents the very recent (since 2013 June) ejection of further AC-forming material. If this is the case the dust mass is $\sim 2.6 \times 10^{-14} \mathrm{M}_{\odot}$ (again for the case of AC), far lower than the earliest dust mass deduced by Evans et al. (2020) $\left(\sim 5 \times 10^{-10} \mathrm{M}_{\odot}\right)$ following the 1998 dust ejection event. Alternatively, Component 2 may be a small dust "cloud", as defined by Hinkle et al. (2020), produced by a single brief ejection event, and which may not grow any further.

Evans et al. (2020) suggested that a dust component having temperature $437 \mathrm{~K}$ in 2014 March, and $411 \mathrm{~K}$ in 2016 July, pointed to renewed mass-loss and dust formation sometime in the period 2008-2014, when the dust mass from this formation event was a few $\times 10^{-8} \mathrm{M}_{\odot}$. The dust we see now at $624 \mathrm{~K}$ (Fig. 2b) is very likely from the same ejection event that produced the $550 \mathrm{~K}$ dust seen by Hinkle \& Joyce in 2013. This indicates that there has been further mass-loss and dust formation between 2013 and 2020. Our identification of Component 2 as yet another, more recent, dust formation event demonstrates that, as far as dust production is concerned, SO continues to be active. Further observa- 


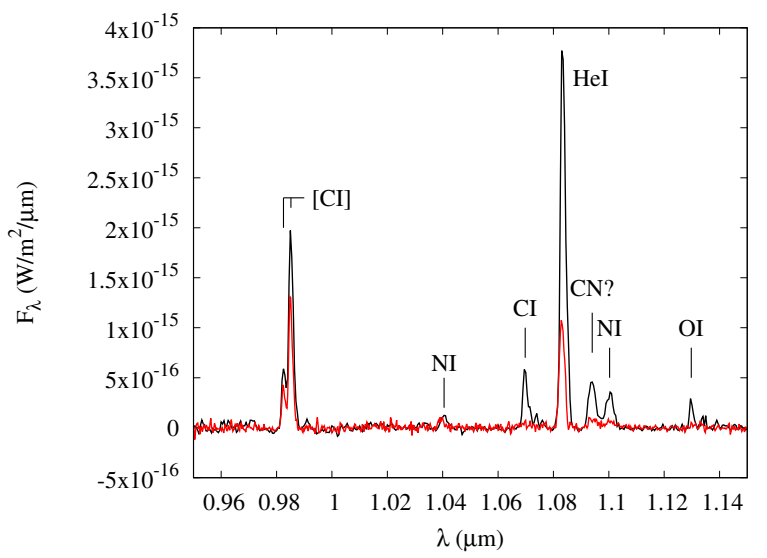

Figure 5. Difference between the emission spectrum in 2013 (red; Hinkle \& Joyce 2014) and in 2020 (black; this work) in the 0.95$1.15 \mu \mathrm{m}$ region. The continuum has been subtracted from both spectra to highlight the difference in the emission lines.

tions are required to verify whether Component 2 is indeed a post-2013 dust formation event, in which case the dust temperature will show clear evidence of declining.

We conclude that the stellar remnant remains unseen and that SO is still puffing out clouds of soot.

\subsection{The emission lines}

Numerous emission lines are present in the 2020 September spectrum, some of which were reported by Hinkle \& Joyce (2014). With the exception of the He I line at $2.059 \mu \mathrm{m}$, these lie in the $0.87-1.7 \mu \mathrm{m}$ region; this portion of the spectrum is shown in Fig. 3 . Each line has been fitted with a gaussian with respect to the adjacent linear continuum; the line centres, fluxes, and the proposed identifications are given in Table 1 The line fluxes generally increased between 2013 June and 2020 September, as illustrated for the $0.95-1.15 \mu \mathrm{m}$ region in Fig. 5 .

Most of the lines arise from transitions in which the upper level is only a few $\mathrm{eV}$ above the ground; the only exceptions are the He I lines, which arise from levels $\sim 20 \mathrm{eV}$ above ground. This is significant in view of the expectation that the central star has an effective temperature $T_{*} \simeq 10^{5} \mathrm{~K}$ and luminosity $L_{*} \sim 10^{3.8} \mathrm{~L}_{\odot}$ (see above). This is explored in Section 3.3 below.

\subsubsection{The O I lines}

The O I lines at $0.8448 \mu \mathrm{m}\left({ }^{3} \mathrm{P}-{ }^{3} \mathrm{~S}^{o}\right)$ and $1.1290 \mu \mathrm{m}$ $\left({ }^{3} \mathrm{D}_{3}^{o}-{ }^{3} \mathrm{P}_{2}\right)$ are both prominent in Fig. 3 however the $1.3168 \mu \mathrm{m}$ line $\left({ }^{3} \mathrm{~S}_{1}-{ }^{3} \mathrm{P}_{2}\right)$ is not detected. In most stellar environments this would suggest that continuum fluorescence is unlikely to play a significant role in the excitation of the first two of the above $\mathrm{O}$ I lines (e.g., Mathew et al. 2018). The $7002 \AA$ and $7254 \AA$ O I lines, which would also be expected if continuum fluorescence were important (Rodríguez-Ardila et al. 2002), were not present in an optical spectrum of SO obtained in 2013 van Hoof et al. 2015a). Also, if recombination were significant, the $7990 \AA$ line, the lower level of which is the upper level of the $0.8448 \mu \mathrm{m}$ line, is expected to be present; but this line was also absent in the 2013 optical spectrum (van Hoof et al. 2015a). Although there is no near-simultaneous red-NIR spectroscopy, we are led to conclude that continuum fluorescence and recombination are unlikely contributors to the excitation of $\mathrm{O}$ I.

This leaves collisional excitation (by electrons or shocks) and Ly- $\beta$ (Bowen) fluorescence (which arises from a close coincidence between the wavelength of $\mathrm{Ly}-\beta$, $1025.7222 \AA$ and that of the ${ }^{3} \mathrm{P}-{ }^{3} \mathrm{D}^{o}$ transition in $\mathrm{O}$, $1025.76 \AA$ ), as possibilities (e.g., Srivastava et al. 2016). However the presence of Ly- $\beta$ photons in a $\mathrm{H}$-deficient environment is clearly problematic, and it is unlikely that such photons will have remained in the circumstellar environment since before the $\mathrm{H}$-deficient phase. While the role of $\mathrm{Ly}-\beta$ in the case of SO might be taken by He II $6-2(\lambda=1025.273 \AA)$, the upper level of this transition is at $50 \mathrm{eV}$, in stark contrast to the relatively low excitation seen in the GNIRS spectrum (see Table 1). In any case there are no other HeII lines in either the optical van Hoof et al. 2015a) or the NIR spectrum presented here. Furthermore, there are no other plausible transitions in other ions that have wavelengths close to that of the ${ }^{3} \mathrm{P}-{ }^{3} \mathrm{D}^{\circ}$ transition in $\mathrm{O}$ I.

We therefore consider collisional excitation to be the most likely excitation mechanism, but even this mechanism is not without difficulty. For electron excitation, the expected flux ratio $I(1.1290 \mu \mathrm{m}) / I(0.8448 \mu \mathrm{m})$ has been calculated by Bhatia \& Kastner (1995), to be $\lesssim 0.03$ for a wide range of electron temperatures $(5000 \mathrm{~K}-100000 \mathrm{~K})$ and densities $\left(10^{4} \mathrm{~cm}^{-3}-10^{12} \mathrm{~cm}^{-3}\right)$, far lower than the (dereddened) value in $\mathrm{SO}, \simeq 0.75$. On the other hand, there may be some evidence for electron collisional excitation of $\left[\mathrm{C}_{\mathrm{I}}\right]$ lines (see Section 3.2 .2 below), and collisional excitation of He I $1.083 \mu \mathrm{m}$ line in SO was suggested by Eyres et al. (1999). Collisional excitation in shocks might also be a possibility, and has been invoked by van Hoof et al. (2007) to account for the decline in emission line fluxes (Section 3.2.2).

Previous investigations of excitation mechanisms have been in the context of environments in which abundances are, even if not solar, not too far removed therefrom. Excitation mechanisms need to be re-examined for environments in which hydrogen is severely deficient.

\subsubsection{The $[C \mathrm{I}]$ lines.}

The [C I] lines at $0.8729,0.9824$ and $0.9850 \mu \mathrm{m}$ (also reported by van Hoof et al. 2007, 2015a) are of particular interest. The two longest wavelength lines (also observed by Hinkle \& Jovce 2014, who did not report the $0.8729 \mu \mathrm{m}$ line) originate from the same ${ }^{1} \mathrm{D}_{2}$ level and, combined with the shortest wavelength line, provide useful constraints on the emitting gas.

At electron temperature $T_{e}=10^{4} \mathrm{~K}$, the critical electron density above which the upper ${ }^{1} \mathrm{D}_{2}$ level is mostly collisionally, rather than radiatively, de-excited is $n_{e}=$ $1.6 \times 10^{4} \mathrm{~cm}^{-3}$ (Liu et al. 1995). Liu et al. also find that the flux ratio $I(0.9824 \mu \mathrm{m}+0.9850 \mu \mathrm{m}) / I(0.8729 \mu \mathrm{m})$ is a potential pointer to the excitation mechanism; we note that this ratio is essentially independent of the assumed reddening as the lines are close in wavelength. We find this flux ratio to have the value $\simeq 9.5$ in the 2020 September spectrum (Fig. [3 and Table 11). Hinkle \& Jovce (2014) found fluxes of $5.9 \times 10^{-19} \mathrm{~W} \mathrm{~m}^{-2}$ and $2.4 \times 10^{-18} \mathrm{~W} \mathrm{~m}^{-2}$ in the $0.9824 \mu \mathrm{m}$ and $0.9850 \mu \mathrm{m}$ lines, respectively, in 2013 
June; a re-examination of their 2013 spectrum suggests that the $0.8729 \mu \mathrm{m}$ line may be weakly present, with a flux of $2.1[ \pm 0.5] \times 10^{-19} \mathrm{~W} \mathrm{~m}^{-2}$, but in any case the upper limit on the flux ratio is $\sim 14$.

Figure 2 of Liu et al. (1995) shows the parameter space for the flux ratio, $n_{e}$ and $T_{e}$. Assuming $T_{e}=10^{4} \mathrm{~K}$, the above two values of the flux ratio seem inconsistent with radiative recombination. On the other hand they seem consistent with collisional excitation by electron impacts if the electron density increased from $\sim 10^{3} \mathrm{~cm}^{-3}$ in 2013 to $\sim 10^{4} \mathrm{~cm}^{-3}$ in 2020. The implied rise in $n_{e}$ may be linked to the increased density in the region where the "Component 2 " dust has formed. Given the critical density for collisional deexcitation from the ${ }^{1} \mathrm{D}_{2}$ level, we might expect the $0.9824 \mu \mathrm{m}$ and $0.9850 \mu \mathrm{m}$ lines to be quenched if $n_{e}$ continues to rise. van Hoof et al. (2007) have argued that the decline in the emission line fluxes is inconsistent with photo-ionisation, but is consistent with excitation in a shock, of uncertain origin, that occurred at some time prior to 2001, after which the gas cooled and recombined.

\subsubsection{The HeI lines}

The He I triplet at $1.0833 \mu \mathrm{m}$ is the strongest emission feature in the spectrum in 2013 and 2020, and increased in strength by a factor of $\sim 3.5$ between the two epochs (see Fig. 5). A weak P Cygni profile in the He I line at $2.0589 \mu \mathrm{m}$ appears to be present in both 2013 and 2020 spectra (see Fig. 6); the absorption appears to be broad and centered at $\sim-700 \mathrm{~km} \mathrm{~s}^{-1}$. This value is similar to those deduced for SO in mid-1998 (Evans et al., to be submitted), and of the same order as determined by Hinkle et al. (2020) for the [C I] lines (see Section 3.2.2). There is no obvious P-Cygni profile in the HeI $1.083 \mu \mathrm{m}$ line. This is not unexpected as details of the radiative transfer in each of these two lines is different.

The dereddened flux ratio $I(1.0833 \mu \mathrm{m}) / I(2.0589 \mu \mathrm{m})$ $\simeq 32$. This is close to the value $(30-33$; see Benjamin et al. 1999) for a gas having electron temperature $10^{4} \mathrm{~K}$ and electron density in the range $10^{4}-10^{6} \mathrm{~cm}^{-3}$; furthermore this line ratio excludes values of $n_{e} \lesssim 10^{4} \mathrm{~cm}^{-3}$. This seems consistent with the $n_{e}$ values implied by the [C I] lines (see Section 3.2.2). This suggests that the He I lines do not suffer significant circumstellar extinction, consistent with a geometry in which the HeI emission arises in jets that are perpendicular to the dust disc (Hinkle et al. 2020) and the lines of sight to the jets do not pass through the dust disc.

\subsubsection{Molecular features}

Evres et al. (1998) reported NIR spectra of SO shortly after its VLTP. The Ballik-Ramsay (1963) $\mathrm{C}_{2}$ bands $\left(A^{\prime 2} \Sigma_{g}^{-}-\right.$ $\left.X^{\prime 3} \Pi_{u}\right)$ at $1.4494 \mu \mathrm{m}(1-2), 1.5063 \mu \mathrm{m}(2-3), 1.7762 \mu \mathrm{m}(0-$ $0), 1.8326 \mu \mathrm{m}(1-1)$ were prominent in absorption, as were the first overtone CO bands. Eyres et al. (1998) also reported that the red system of the $A^{2} \Pi-X^{2} \Sigma$ electronic transition in $\mathrm{CN}$ was very prominent in absorption at $1.1 \mu \mathrm{m}$ $\left(\nu^{\prime}=0 \rightarrow \nu^{\prime \prime}=0\right)$, and was the strongest of the $\mathrm{CN}$ bands in their spectral range.

Much later in the evolution of the VLTP, Evans et al. (2006) found the presence of small molecules (HCN, acety-
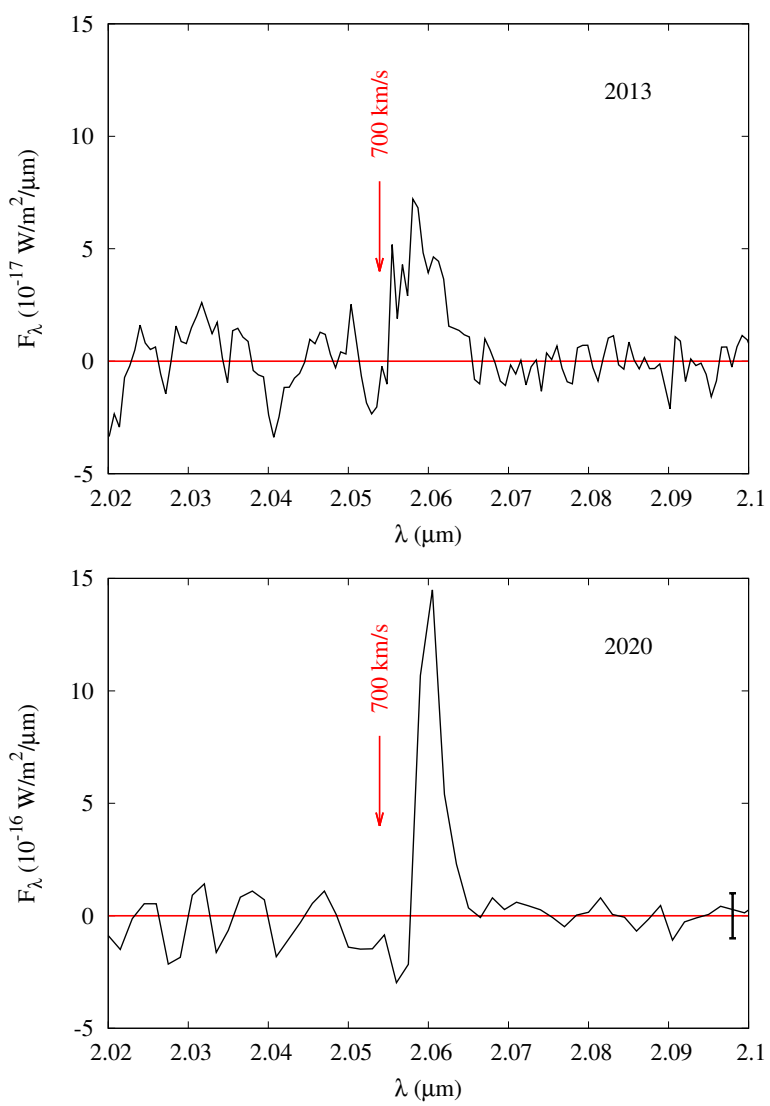

Figure 6. Possible P Cyg profiles in He I $2.058 \mu \mathrm{m}$; upper panel, 2013, lower panel 2020. The error bar in the 2020 data, derived from the fluctuations in the data points in the intervals 2.02 $2.045 \mu \mathrm{m}$ and $2.075-2.10 \mu \mathrm{m}$, is $\pm 1 \sigma$. Note that, at the resolution of this spectrum, the telluric transmission, which contains numerous strong and narrow absorption lines of $\mathrm{CO}_{2}$, does not resolve individual $\mathrm{CO}_{2}$ lines from one another and that the observed $\mathrm{CO}_{2}$ absorption reaches a maximum depth of only $\sim 25 \%$ below the continuum.

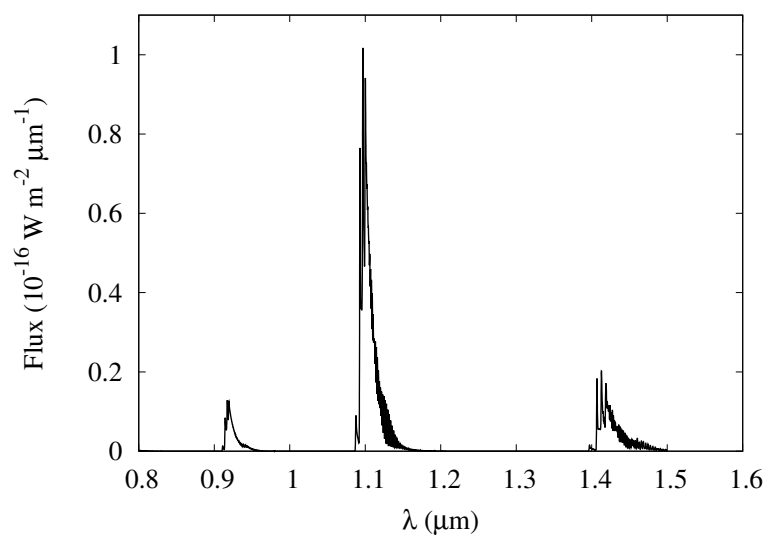

Figure 7. Calculated emission from CN at $1000 \mathrm{~K}$, using CN data from Brooke et al. (2014); main emission feature is at $1.09 \mu \mathrm{m}$. The theoretical CN spectrum has not been convolved with the instrumental resolution. See text for discussion. 
lene, polyynes) in the mid-IR. Around the same time (20032004) Worters et al. (2009) found that the CO fundamental was prominent in absorption against the dust shell.

van Hoof et al. (2018) reported a complex of some five emission lines around $0.9 \mu \mathrm{m}$, which had been emerging since 2013; they tentatively identified some of the latter features with the $(1,0)$ and $(0,0)$ transitions in the red system of the $A^{2} \Pi-X^{2} \Sigma$ electronic transition in $\mathrm{CN}$.

We find no evidence for emission or absorption by $\mathrm{CO}$ or $\mathrm{C}_{2}$ over the entire wavelength range in the 2020 September observation. The case for or against $\mathrm{CN}$ is less clear-cut. We note that, while all the stronger lines in the $0.8-2.5 \mu \mathrm{m}$ range are robustly identified, there are prominent features that remain unidentified, around $1.0940 \mu \mathrm{m}$ and $1.4075 \mu \mathrm{m}$; these are tantalisingly close to some of the CN $\Delta v=0$ and $\Delta v=1$ band-head positions (Evres et al. 1998). Indeed the $1.09 \mu \mathrm{m}$ feature was tentatively identified with $\mathrm{CN}$ by Hinkle et al. (2020).

Fig. 7 shows the expected emission from optically thin ${ }^{12} \mathrm{CN}$ in Local Thermal Equilibrium (LTE) at $1000 \mathrm{~K}$, calculated using molecular data from Brooke et al. (2014). However the situation is complicated by (a) the likelihood that the environment of SO is highly non-LTE (see, e.g., Liu. Dalgarno \& Lepp 1992, for the effects of non-LTE on CO emission), (b) the certain presence of ${ }^{13} \mathrm{CN}$, in view of the low ${ }^{12} \mathrm{C} /{ }^{13} \mathrm{C}$ ratio, and the fact that optical depth effects are not included in our simple model. A simple comparison is not feasible without detailed modelling along these lines. There are no other plausible candidates for these features.

An emission feature at $1.09 \mu \mathrm{m}$ was detected in the NIR spectrum of the peculiar helium nova V445 Pup by Ashok \& Baneriee (2003), who attributed it to C II; however other expected $\mathrm{C}$ II lines were not obviously present. This object, like SO, displayed numerous He and $\mathrm{C}$ I lines, but none of hydrogen (Ashok \& Baneriee 2003). Both SO and V445 Pup have C-rich, H-deficient environments.

\subsection{Where is the hot central star?}

The evolutionary track given in Haiduk et al. (2005) suggests that SO's central star should, at the time of the 2020 observation, have had an effective temperature close to $10^{5} \mathrm{~K}$. However, as noted in Section 3.2 the emission lines - with the exception of He I - arise in upper levels having relatively low excitation. Moreover, all the identified species are neutral.

This inevitably raises the question: is the expected hot remnant of the 1996 VLTP present? One might reasonably expect a source at $10^{5} \mathrm{~K}$ to ionise the surrounding gas, but there is no evidence for a higher degree of ionisation and excitation. Even at slightly lower temperatures, for example 30000-50000 K (typical of the central star temperatures of a large number of planetary nebulae), a higher degree of excitation would be expected in the NIR spectrum (e.g., Rudv et al. 2001). A plausible explantion is that ionising radiation does not reach the emitting gas seen in Fig. 3 because of internal extinction by the dust. However the dust geometry takes the form of a disc (Chesneau et al. 2009), and some hard radiation must emerge along the disc axis. Indeed, that there is emitting material, which is extended, along this axis is demonstrated by the observations of Hinkle et al. (2020) (see also Section 4 below): in the presence of a hot $\left(\sim 10^{5} \mathrm{~K}\right)$
Table 2. Helicentric radial velocities of peak emission as determined from the HeI and [C I] profiles in Fig. 8

\begin{tabular}{|c|c|c|c|c|c|}
\hline $\begin{array}{c}\text { Offset } \\
\text { along slit }\left({ }^{\prime \prime}\right)\end{array}$ & $\begin{array}{l}\text { O!' } 90 \\
\text { NNE }\end{array}$ & $\begin{array}{l}0 . \prime 45 \\
\text { NNE }\end{array}$ & $\begin{array}{c}0^{\prime \prime} 0 \\
\text { Centre }\end{array}$ & $\begin{array}{l}0 . ! 45 \\
\text { SSW }\end{array}$ & $\begin{array}{l}0 . \\
\text { SSW }\end{array}$ \\
\hline $\begin{array}{l}\text { He I velocity }\left(\mathrm{km} \mathrm{s}^{-1}\right) \\
{\left[\mathrm{C}_{\mathrm{I}}\right] \text { velocity }\left(\mathrm{km} \mathrm{s}^{-1}\right)}\end{array}$ & $\begin{array}{l}+321 \\
+217\end{array}$ & $\begin{array}{l}+210 \\
+169\end{array}$ & $\begin{array}{l}-50 \\
-61\end{array}$ & $\begin{array}{l}-125 \\
-151\end{array}$ & $\begin{array}{l}-111 \\
-195\end{array}$ \\
\hline
\end{tabular}

source, there would surely be highly ionised material, such as He II, in this region.

Another explanation is that material close to the central star, and located within the dust disc, is indeed highly ionised. However this ionised material would be visible at long wavelengths even through $\sim 10 \mathrm{mag}$ of visual extinction. For example, Asplund et al. (1999) determined the neon abundance in SO's photosphere to be $\sim 1.4$ dex higher than solar, so we would expect to see evidence of higher ionisation states of Ne, such as [Ne II] $(12.814 \mu \mathrm{m})$, [Ne III] $(15.555 \mu \mathrm{m})$ and $[\mathrm{Nev}](14.322 \mu \mathrm{m})$, at which wavelengths extinction by the dust would be much reduced. However none of these lines are present in Spitzer IRS and SOFIA spectra of SO (Evans et al. 2006, 2020), while the first two are present in the Spitzer IRS spectrum of the VLTP V605 Aql (Evans et al. 2006).

The implication is that there is no hot star; at present the temperature of the "photosphere" of the central object of $\mathrm{SO}$ is much less than $10^{5} \mathrm{~K}$, and that the evolutionary models, at least for SO, may need revisiting.

\section{EXTENDED EMISSION}

Hinkle \& Jovce (2014) found that emission in the He I $1.0833 \mu \mathrm{m}$ line in 2010 September had radial velocities in the range $\sim-800 \mathrm{~km} \mathrm{~s}^{-1}$ to $\sim+200 \mathrm{~km} \mathrm{~s}^{-1}$; they further found that the emission was extended, by about $\sim 1$ 1.9 to zero intensity. They found that [C I] $0.9850 \mu \mathrm{m}$ was also extended, but that its range of radial velocities was significantly different $\left(\sim-400 \mathrm{~km} \mathrm{~s}^{-1}\right.$ to $\left.\sim+300 \mathrm{~km} \mathrm{~s}^{-1}\right)$ from that of He I. Recent observations (Hinkle et al. 2020) show that the He I emission region had expanded during the period 2010-2019, and has a bipolar structure with a dark lane at position angle $\simeq 120^{\circ}$, consistent with that of the dense dusty disc detected by Chesneau et al. (2009), and with that of the old planetary nebula at whose centre SO is located.

Our 2020 September data (Fig. 8) also show that the He I and [C I] $0.9850 \mu \mathrm{m}$ emission are extended. At $0 . .9 \mathrm{NNE}$ of the central star, the radial velocity of peak He I emission peaks is $\sim+300 \mathrm{~km} \mathrm{~s}^{-1}$, but significant emission is present up to $\sim+900 \mathrm{kms}^{-1}$. There is no evidence for emission beyond $\sim 1^{\prime \prime}$ from the central object at this position angle. Hinkle et al. (2020) found that the He I $1.0833 \mu \mathrm{m}$ and [C I] $0.9850 \mu \mathrm{m}$ lines were resolved in a 2015 spectrum, the linewidths in their 2015 spectrum implied an expansion velocity of $\sim 500 \mathrm{~km} \mathrm{~s}^{-1}$.

The emission to the SSW is much weaker and is blueshifted, with the peak emission at heliocentric radial velocity of $\sim-200 \mathrm{~km} \mathrm{~s}^{-1}$. Similar emission profiles are seen in the [C I] $0.9824 / 0.9850 \mu \mathrm{m}$ lines (note that, in Fig. 8 , the $0.9824 \mu \mathrm{m}[\mathrm{C} \mathrm{I}]$ line appears as a feature $\sim 800 \mathrm{~km} \mathrm{~s}^{-1}$ blueward of the stronger $0.9850 \mu \mathrm{m}$ line). We have determined 

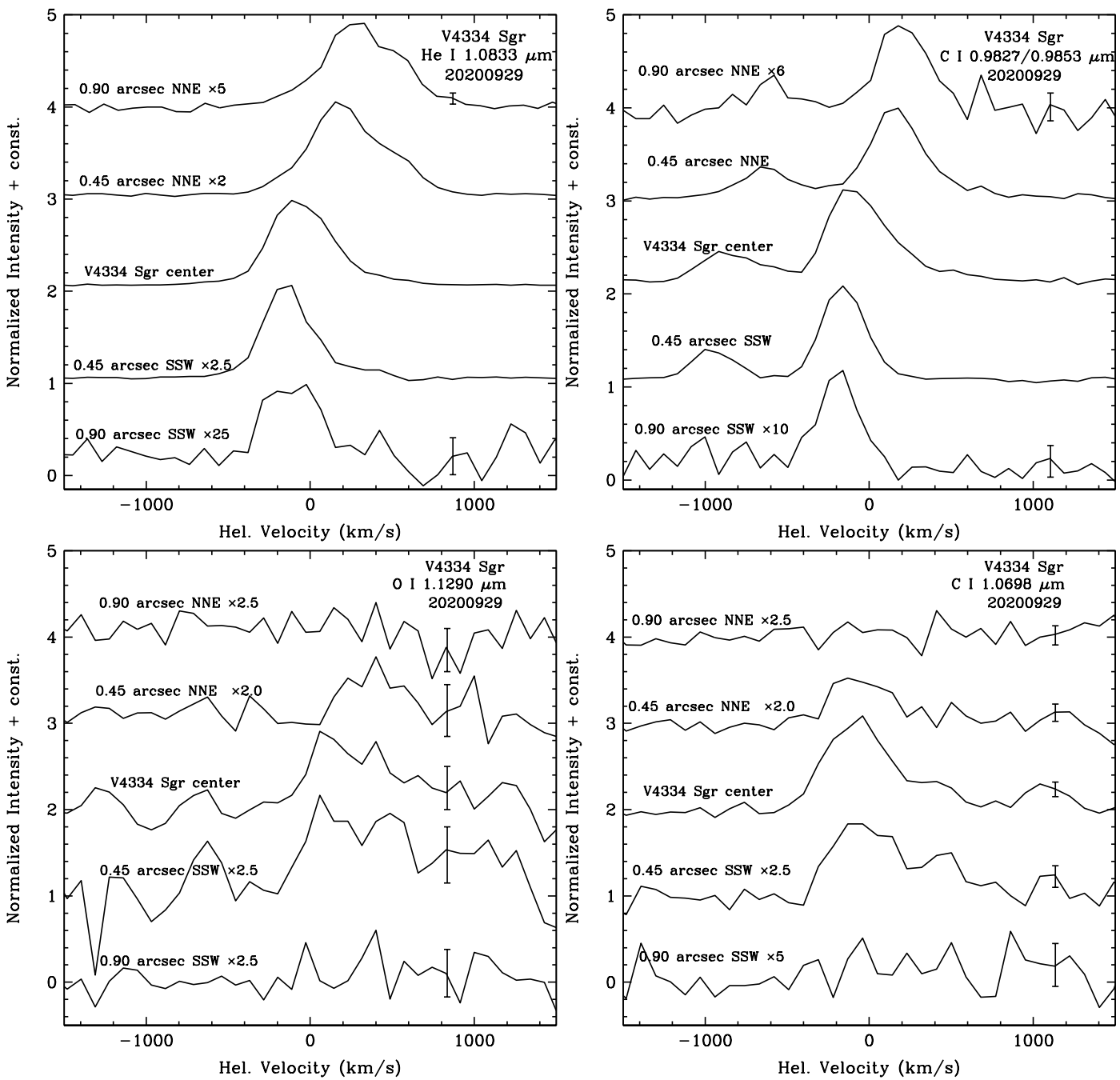

Figure 8. Top left: the He I $1.0833 \mu \mathrm{m}$ profile at five positions along the slit. Top right: same for the [C I] $0.9824 / 0.9850 \mu \mathrm{m}$ lines; the heliocentric velocity in this case is that for the stronger $0.9850 \mu \mathrm{m}$ line; the shorter wavelength line appears as a feature at $-796 \mathrm{~km} \mathrm{~s}^{-1}$. Each spectrum is the sum of three adjacent rows (covering 0!' 45). Bottom left: same for O I $1.1290 \mu \mathrm{m}$. Bottom right: same for C I $1.0688 \mu \mathrm{m}$. The error bars are $\pm 1 \sigma$; the error bars are negligible for some of the higher signal-to-noise spectra.

the peak heliocentric velocities for each of the He I $1.0833 \mu \mathrm{m}$ and [C I] $0.9850 \mu \mathrm{m}$ lines in the top two panels of Fig. 8 The velocities of peak emission are given in Table 2

There is marginal evidence for extended emission, out to $0^{\prime \prime} .45$, in the $\mathrm{O}$ I line at $1.1290 \mu \mathrm{m}$, and in the $\mathrm{C}$ I $1.0688 \mu \mathrm{m}$ line. The signal-to-noise ratio for these two features is rather low (see Fig. 8).

Although the signal-to-noise ratios in some of the line profiles are rather low, Fig. 8] suggests that the line profiles of the $\mathrm{He}$ I, $\mathrm{O}$ I and $\mathrm{C}$ I lines are broadly similar, indicating that they arise in the same region. This would be consistent with our conclusion (see Section 3.2.1) that they suffer similar extinctions.

\section{CONCLUSIONS}

We have presented a new $0.8-2.5 \mu \mathrm{m}$ spectrum of the Very Late Thermal Pulse object V4334 Sgr (Sakurai's Object). We conclude that: (i) the effective temperature of the stellar remnant is unlikely to be as high as that implied by current evolutionary models;

(ii) the large increase in continuum flux density near $1 \mu \mathrm{m}$ is due to a very recent episode of extremely hot, likely amorphous carbon, dust formation;

(iii) the stellar component remains unseen;

(iv) the HeI lines suffer negligible circumstellar extinction, suggesting that they arise in jets having lines of sight that do not pass through the dust disc;

(v) the relative intensities of the $\left[\mathrm{C}_{\mathrm{I}}\right]$ NIR lines suggest a rise in the electron density from 2013 to 2020;

(vi) there is some evidence for emission by the $\mathrm{CN}$ radical; there is no evidence for emission by other molecules in the $0.82-2.5 \mu \mathrm{m}$ range.

In view of the continuing rapid spectral evolution of Sakurai's Object, further IR observations of it in this and other wavelength bands are strongly encouraged 


\section{ACKNOWLEDGMENTS}

This paper is based on observations obtained at the international Gemini Observatory, a program of NSF's NOIRLab, which is managed by the Association of Universities for Research in Astronomy (AURA) under a cooperative agreement with the National Science Foundation, on behalf of the Gemini Observatory partnership: the National Science Foundation (United States), National Research Council (Canada), Agencia Nacional de Investigación y Desarrollo (Chile), Ministerio de Ciencia, Tecnología e Innovación (Argentina), Ministério da Ciência, Tecnologia, Inovações e Comunicações (Brazil), and Korea Astronomy and Space Science Institute (Republic of Korea).

Data were also obtained under IRTF programs 2020A001 and 2020A-010. The Infrared Telescope Facility is operated by the University of Hawaii under contract 80HGTR19D0030 with the National Aeronautics and Space Administration.

DPKB is supported by a CSIR Emeritus Scientist grantin-aid and is being hosted by the Physical Research Labora-

tory, Ahmedabad. RDG was supported by the United States Airforce. CEW was supported by USRA/SOFIA contract SOF 07-0027 (NASA prime award NNA17BF53C).

\section{DATA AVAILABILITY}

The raw data in this paper that were obtained at the Gemini Observatory are available from the Gemini Observatory Archive, https://archive.gemini.edu/. 


\section{REFERENCES}

Ashok N. M., Banerjee D. P. K., 2003, A\&A, 409, 1007

Asplund M., Lambert D. L., Kipper T., Pollacco D., Shetrone M. D., 1999 A\&A, 343, 507

Ballik E. A., Ramsay D. A., 1963, ApJ, 137, 61

Benjamin R. A., Skillman E. D., Smits D. P., 1999, ApJ, 514,307

Bhatia A. K., Kastner S.O., 1995, ApJS, 96, 325

Brooke J. S. A., Ram R. S., Western C.M., Li G., Schwenke D. W., Bernath P. F., 2014, ApJS, 210, 23

Chesneau O., et al., 2009, A\&A, 493, L17

Clayton G. C., 2012, JAAVSO, 40, 539

Dürbeck H. W., et al., 2000, AJ, 119, 2360

Elias J. H., Joyce R. R., Liang M., Muller G. P, Hileman, E. A., George J. R., 2006, Proc. SPIE, 6269, 138

Evans A., et al., 2006, MNRAS, 373, L75

Evans A., et al., 2017, MNRAS, 446, 4221

Evans A., et al., 2020, MNRAS, 493, 1277

Eyres S. P. S., Evans A., Geballe T. R., Salama A., Smalley B., 1998, MNRAS, 298, L37

Eyres S. P. S., Smalley B., Geballe T. R., Evans A., Asplund M., Tyne V. H., 1999, MNRAS, 307, L11

Gall C., Hjorth J., Watson D., Dwek E., Maund J. R., Fox O., Leloudas G., Malesani D., Day-Jones A. C., 2014, Nature, 511, 326

Hajduk M., et al. 2005, Science, 308, 231

Hanner M., 1988, in Infrared Observations of Comets Halley and Wilson and Properties of the Grains, NASA Conference Publication 3004, ed M. S. Hanner, p. 22, Washington DC

Haris K., Kramida A., 2017, ApJS, 233, 16

Harrison T. E., Stringfellow G. S., 1994, ApJ, 437, 827

Hinkle K. H., Joyce R. R., 2014, ApJ, 785, 146

Hinkle K. H., Joyce R. R., Matheson T., Lacy J. H., Richter M. J., 2020, ApJ, 904, 34

Holbrook J. C., Temi P., 1998, ApJ, 496, 280

Liu W., Dalgarno A., Lepp S., 1992, ApJ, 396, 679

Liu X.-W., Barlow M. J., Danziger I. J., Clegg R. E. S., 1995, MNRAS, 273, 47

Lodders K., Fegley B., 1995, Meteoritics, 30, 661

Lynch D. K., et al., 2008, AJ, 136, 1815

Mathew B., et al., 2018, ApJ, 857, 30

Pendleton Y. J., Tielens A. G. G. M., Werner M. W., 1990, ApJ, 349, 107

Rayner J. T., et al., 2003, PASP, 115, 362

Rodríguez-Ardila A., Viegas S. M., Pastoriza M. G., Prato L., Donzelli C. J., 2002, ApJ, 572, 94

Rudy R. J., Lynch D. K., Mazuk S., Puetter R. C., Dearborn D. S. P., 2001, AJ, 121, 632

Sellgren K., 1984, ApJ, 277, 623

Shortridge K., et al., 2004, Starlink Project, Starlink User Note 86.21

Srivastava M. K., Banerjee D. P. K., Ashok N. M., Venkataraman V., Sand D., Diamond T., 2016, MNRAS, 462, 2074

Tody D., 1989, SPIE, 627, 733

Tyne V. H., Evans A., Geballe T. R., Eyres S. P. S., Smalley B., Dürbeck H. W., 2002, MNRAS, 334, 875

van Hoof P. A. M., et al., 2007, A\&A, 471, L9

van Hoof P. A. M., Hajduk M., Zijlstra A. A., Herwig F., Van de Steene G. C., Kimeswenger S., Evans A., 2008, in Hydrogen-Deficient Stars, Astronomical Society of the Pacific Conference Series, vol. 391, p. 155, eds K. Werner and T. Rauch, San Francisco

van Hoof P. A. M., Kimeswenger S., Van de Steene G. C., Zijlstra A. A., Hajduk M., Herwig F., 2015a, in 19th European Workshop on White Dwarfs, Astronomical Society of the Pacific Conference Series, vol. 493, p. 95, eds P. Dufour, P. Bergeron, G. Fontaine, San Francisco

van Hoof P. A. M., Van de Steene G. C., Kimeswenger S., Zijlstra A. A., Hajduk M., Herwig F., 2015b, in The Physics of Evolved Stars: A conference dedicated to the memory of Olivier Chesneau, European Astronomical Society Publication Series, vol. 71-72, p. 287, eds E. Lagadec, F. Millour, T. Lanz, EDP Sciences, Les Ulis

van Hoof P. A. M., 2018, Galaxies, 6, 63

van Hoof P. A. M., Kimeswenger S., Van de Steene G. C., Avison A., Zijlstra A. A., Guzman-Ramirez L., Herwig F., Hajduk M., 2018, Galaxies, 6, 36, Special Issue Asymmetric Planetary Nebulae VII, eds Q. A. Parker, N. Soker, Basel, Switzerland

Van de Steene G. C., van Hoof P. A. M., Kimeswenger S., Zijlstra A. A., Avison A., Guzman-Ramirez L., Hajduk M., Herwig F., 2016, in Planetary Nebulae: MultiWavelength Probes of Stellar and Galactic Evolution, Proceedings of IAU Symposium 323, eds X. Liu, L. Stanghellini, A. Karakas, p. 380, Cambridge University Press, Cambridge, UK

Worters H. L., Rushton M. T., Eyres S. P. S., Geballe T. R., Evans A., 2009, MNRAS, 393, 108 
This paper has been typeset from a $\mathrm{T}_{\mathrm{E}} \mathrm{X} / \mathrm{LT}_{\mathrm{E}} \mathrm{X}$ file prepared by the author. 